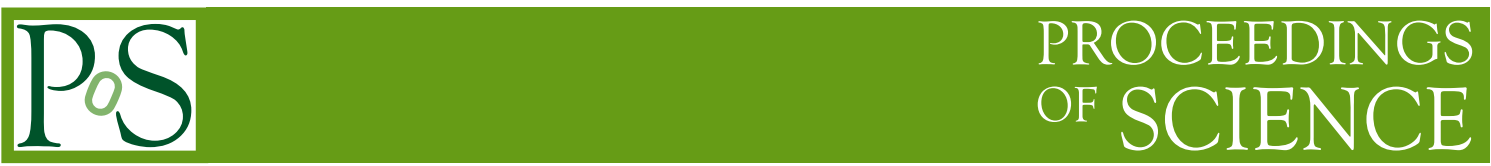

\title{
Hadron Spectroscopy in COMPASS
}

\author{
Boris Grube ${ }^{* \dagger}$ on behalf of the COMPASS collaboration \\ CERN, Geneva, Switzerland \\ On leave of absence from \\ Physik-Department E18, Technische Universität München, Garching, Germany \\ E-mail: bgrubeatum.de
}

The COmmon Muon and Proton Apparatus for Structure and Spectroscopy (COMPASS) is a multi-purpose fixed-target experiment at the CERN Super Proton Synchrotron (SPS) aimed at studying the structure and spectrum of hadrons.

In the naïve Constituent Quark Model (CQM) mesons are bound states of quarks and antiquarks. QCD, however, predict the existence of hadrons beyond the CQM with exotic properties interpreted as excited glue (hybrids) or even pure gluonic bound states (glueballs). One main goal of COMPASS is to search for these states. Particularly interesting are so called spin-exotic mesons which have $J^{P C}$ quantum numbers forbidden for ordinary $q \bar{q}$ states.

Its large acceptance, high resolution, and high-rate capability make the COMPASS experiment an excellent device to study the spectrum of light-quark mesons in diffractive and central production reactions up to masses of about $2.5 \mathrm{GeV} / \mathrm{c}^{2}$. COMPASS is able to measure final states with charged as well as neutral particles, so that resonances can be studied in different reactions and decay channels.

During 2008 and 2009 COMPASS acquired large data samples using negative and positive secondary hadron beams on $\ell \mathrm{H}_{2}, \mathrm{Ni}$, and $\mathrm{Pb}$ targets. The presented overview of the first results from this data set focuses in particular on the search for spin-exotic mesons in diffractively produced $\pi^{-} \pi^{+} \pi^{-}, \eta \pi, \eta^{\prime} \pi$, and $\pi^{-} \pi^{+} \pi^{-} \pi^{+} \pi^{-}$final states and the analysis of central-production of $\pi^{+} \pi^{-}$pairs in order to study glueball candidates in the scalar sector.

Xth Quark Confinement and the Hadron Spectrum

8-12 October 2012

TUM Campus Garching, Munich, Germany

\footnotetext{
* Speaker.

${ }^{\dagger}$ This work is supported by the German BMBF, the Maier-Leibnitz-Labor der LMU und TU München, the DFG Cluster of Excellence Origin and Structure of the Universe, and CERN-RFBR grant 08-02-91009.
} 


\section{Experimental Setup}

COMPASS is a two-stage high-resolution spectrometer that covers a wide range of scattering angles and particle momenta [1]. Both stages of the spectrometer are equipped with hadronic and electromagnetic calorimeters so that final states with charged as well as neutral particles can be reconstructed. A Ring-Imaging Cherenkov Detector (RICH) in the first stage can be used for particle identification. It is able to separate kaons from pions up to momenta of about $50 \mathrm{GeV} / c$. The target is surrounded by a Recoil Proton Detector (RPD) that measures the time of flight of recoil protons using two scintillator barrels. COMPASS uses the M2 beam line of the SPS which can deliver secondary hadron beams with a momentum of up to $280 \mathrm{GeV} / c$ and a maximum intensity of $5 \cdot 10^{7} \mathrm{~s}^{-1}$. The negative hadron beam that was used for the analyses presented here has a momentum of $190 \mathrm{GeV} / c$ and consists of $97 \% \pi^{-}, 2 \% K^{-}$, and $1 \% \bar{p}$ at the COMPASS target. Two ChErenkov Differential counters with Achromatic Ring focus (CEDAR) upstream of the target are used to identify the incoming beam particles.

\section{Search for Spin-Exotic Mesons in Diffractive Dissociation}

Diffractive dissociation reactions are known to exhibit a rich spectrum of produced intermediate states. In the past several candidates for spin-exotic mesons have been reported in pion-induced diffraction [2]. In diffractive events the beam hadron is excited to some intermediate state $X$ via $t$-channel Reggeon exchange with the target. At $190 \mathrm{GeV} / c$ beam momentum Pomeron exchange is dominant. The $X$ decays into a $n$-body final state which is detected by the spectrometer. The process beam + target $\rightarrow X+$ recoil, where $X \rightarrow h_{1} \ldots h_{n}$, is characterized by two kinematic variables: the square of the total center-of-mass energy, $s$, and the squared four-momentum transfer to the target, $t=\left(p_{\text {beam }}-p_{X}\right)^{2}$. It is customary to use the variable $t^{\prime} \equiv|t|-|t|_{\text {min }}$ instead of $t$, where $|t|_{\min }$ is the minimum value of $|t|$ for a given invariant mass of $X$.

\section{$2.1 \pi^{-} \pi^{+} \pi^{-}$Final States from $\pi^{-}$Diffraction}

A partial-wave analysis (PWA) of $\pi^{-} \pi^{+} \pi^{-}$final states produced in $\pi^{-}$diffraction on a $\mathrm{Pb}$ target in the squared four-momentum transfer range of $0.1<t^{\prime}<1.0(\mathrm{GeV} / c)^{2}$ and extracted from data taken during the pilot run in 2004 showed significant intensity in the spin-exotic $J^{P C}=1^{-+}$ partial wave in the $\rho^{0} \pi^{-}$decay channel [3]. A Breit-Wigner fit yielded resonance parameters consistent with the disputed $\pi_{1}(1600)$ claimed in this channel by other experiments [4].

In 2008 COMPASS has acquired a large data set of the diffractive dissociation reaction $\pi^{-} p \rightarrow$ $\pi^{-} \pi^{+} \pi^{-} p_{\text {slow }}$. The trigger included a beam definition and the RPD, which ensured that the target proton stayed intact and also introduced a lower bound for $t^{\prime}$ of about $0.1(\mathrm{GeV} / c)^{2}$. Diffractive events were enriched by an exclusivity cut around the nominal beam energy. After all cuts the analyzed $\pi^{-} \pi^{+} \pi^{-}$sample contains about $5 \cdot 10^{7}$ events in the range $0.1<t^{\prime}<1.0(\mathrm{GeV} / c)^{2}$.

The PWA approach employs the isobar model [5] in which the decay $X^{-} \rightarrow \pi^{-} \pi^{+} \pi^{-}$is decomposed into a chain of successive two-body decays. The $X^{-}$with quantum numbers $J^{P C}$ and spin projection $M^{\varepsilon}$ is assumed to decay into a $\pi^{+} \pi^{-}$resonance, the so-called isobar, and a bachelor pion. The isobar has spin $S$ and a relative orbital angular momentum $L$ with respect to $\pi_{\text {bachelor }}^{-}$. 

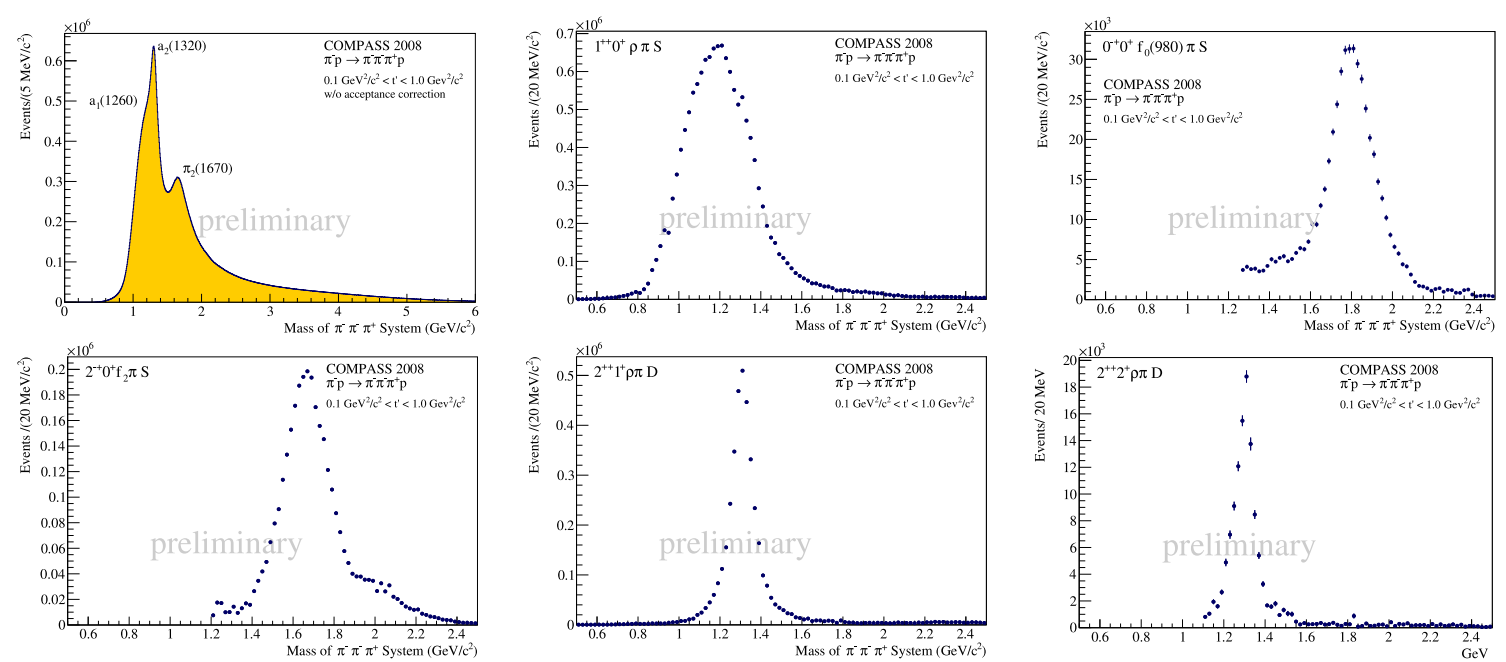

Figure 1: Left and center columns: $\pi^{-} \pi^{+} \pi^{-}$invariant mass spectrum (top left). Intensities of the three major waves: $1^{++} 0^{+}[\rho \pi] S$ wave with the $a_{1}(1260)$ (top center), $2^{++} 1^{+}[\rho \pi] D$ wave with the $a_{2}(1320)$ (bottom center), and $2^{-+} 0^{+}\left[f_{2} \pi\right] S$ wave with the $\pi_{2}(1670)$ (bottom left). Right column: Examples for small waves (note the different $y$ scale): $0^{-+} 0^{+}\left[f_{0}(980) \pi\right] S$ wave with the $\pi(1800)$ (top right) and $2^{++} 2^{+}$ $[\rho \pi] D$ with the $a_{2}(1320)$ (bottom right).

A partial wave is thus defined by $J^{P C} M^{\varepsilon}$ [isobar $] L$, where $\varepsilon= \pm 1$ is the reflectivity which corresponds to the naturality of the exchanged particle in the production process [6].

The spin-density matrix for the chosen set of 52 partial waves plus an incoherent isotropic background wave is determined by unbinned extended maximum likelihood fits performed in $20 \mathrm{MeV} / c^{2}$ wide bins of the three-pion invariant mass $m_{X}$. In these fits no assumption is made on the produced resonances $X^{-}$other than that their production strength is constant within a $m_{X}$ bin. The PWA model includes five $\pi^{+} \pi^{-}$isobars [7]: $(\pi \pi)_{S \text {-wave }}, \rho(770), f_{0}(980), f_{2}(1270)$, and $\rho_{3}(1690)$. They were described using relativistic Breit-Wigner line shape functions including Blatt-Weisskopf barrier penetration factors [8]. For the $\pi^{+} \pi^{-} S$-wave we use the parametrization from [9] with the $f_{0}(980)$ subtracted from the elastic $\pi \pi$ amplitude and added as a separate Breit-Wigner resonance. Mostly natural-parity waves are needed to describe the data. A rank-two spin-density matrix was used in order to account for spin-flip and spin-non-flip amplitudes at the target vertex.

The intensities of the three dominant waves in the $\pi^{-} \pi^{+} \pi^{-}$final state, $1^{++} 0^{+}[\rho \pi] S, 2^{++} 1^{+}$ $[\rho \pi] D$, and $2^{-+} 0^{+}\left[f_{2} \pi\right] S$, are shown in Fig. 1 left and center. They contain resonant structures that correspond to the well-known $a_{1}(1260), a_{2}(1320)$, and $\pi_{2}(1670)$, respectively [7]. That the applied analysis technique is able to cleanly separate even small waves with intensities at the percent level of that of the dominant waves is illustrated in Fig. 1 right. It shows two exemplary waves, $2^{++} 2^{+}$ $[\rho \pi] D$ and $0^{-+} 0^{+}\left[f_{0}(980) \pi\right] S$, which exhibit clear peaks of the $a_{2}(1320)$ (this time with $M=2$ ) and the $\pi(1800)$, respectively.

Figure 2 left shows the intensity of the spin-exotic $1^{-+} 1^{+}[\rho \pi] P$ wave. The bump around $1.2 \mathrm{GeV} / c^{2}$ does not seem to be of resonant nature. It is unstable with respect to changes in the PWA model which hints that it is rather an artifact of the analysis method. On the other hand the peak structure around $1.6 \mathrm{GeV} / c^{2}$ as well as the corresponding rising phase motion with respect to the tail of the $a_{1}(1260)$ in the $1^{++} 0^{+}[\rho \pi] S$ wave (cf. Fig. 2 center) are stable against modifications 

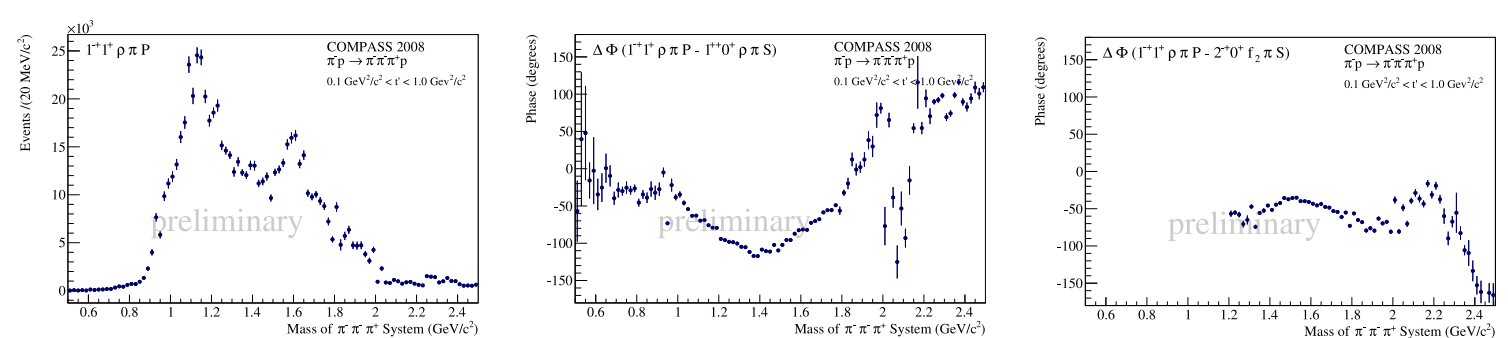

Figure 2: Left: Intensity of the spin-exotic $1^{-+} 1^{+}[\rho \pi] D$ wave. Center: Phase of the $1^{-+}$wave relative to the $1^{++} 0^{+}[\rho \pi] S$ wave. Right: Relative phase with respect to the $2^{-+} 0^{+}\left[f_{2} \pi\right] S$ wave.

of the fit model. As Fig. 2 right shows, the structure is phase locked with the $\pi_{2}(1670)$ in the $2^{-+}$ $0^{+}\left[f_{2} \pi\right] S$ wave. This is consistent with the results obtained from a PWA of the pilot-run data taken with a $\mathrm{Pb}$ target [3]. The interpretation of the $1^{-+}$wave in terms of resonances, however, is still unclear. There seem to be significant contributions from non-resonant Deck-like processes that need to be included into the fit model.

The partial waves also exhibit different $t^{\prime}$ behavior depending on their spin projection $M$ and their resonance contents. This is problematic, since the data cover a rather wide $t^{\prime}$ range from 0.1 to $1.0(\mathrm{GeV} / c)^{2}$ while at the same time the PWA model assumes full coherence of all partial waves. This issue will be addressed by performing a two-dimensional PWA in bins of $m_{X}$ and $t^{\prime}$.

\section{$2.2 \pi^{-} \eta$ and $\pi^{-} \eta^{\prime}$ Final States from $\pi^{-}$Diffraction}

Previous experiments claimed spin-exotic $J^{P C}=1^{-+}$resonances also in the $\pi^{-} \eta[10,11]$ and $\pi^{-} \eta^{\prime}[10,12]$ final states. However, the resonant nature of the observed signals is still controversial [13]. COMPASS performed a PWA of both final states in the diffractive reaction $\pi^{-} p \rightarrow \pi^{-} \eta^{(\prime)} p_{\text {slow }} \rightarrow \pi^{-} \pi^{+} \pi^{-} \gamma \gamma p_{\text {slow }}$ in the kinematic range $0.1<t^{\prime}<1.0(\mathrm{GeV} / c)^{2}$ [14]. The $\eta$ is reconstructed via its decay to $\pi^{+} \pi^{-} \pi^{0}$ where the $\pi^{0}$ goes to a photon pair. In order to reconstruct the $\eta^{\prime}, \eta$ reconstructed in the $\gamma \gamma$ channel are combined with a $\pi^{+} \pi^{-}$pair. The final selected data sample contains about $100000 \pi^{-} \eta$ and $35000 \pi^{-} \eta^{\prime}$ events.

The performed PWA follows previous analyses and includes $S, P$, and $D$ waves with $M \leq 1$ and both natural and unnatural parity exchange. In addition a $4^{++} 1^{+} G$ wave and an incoherent isotropic background wave were included. For both channels the bulk of the intensity is described by the $1^{-+}, 2^{++}$, and $4^{++}$waves with $M=1$ and natural parity exchange.

The left column of Fig. 3 shows the $2^{++} 1^{+}\left(D_{+}\right)$intensity for the two final states. Although the two distributions look rather different they are related to each other by the fact that $\eta$ and $\eta^{\prime}$ are mixtures of the basis states $\eta_{n}=(1 / \sqrt{2})(u \bar{u}+d \bar{d})$ and $\eta_{s}=s \bar{s}$. The partial-wave amplitudes $T_{J}$ of a spin- $J$ resonance with negligible $s \bar{s}$ content decaying into $\pi^{-} \eta$ and $\pi^{-} \eta^{\prime}$ should be related to each other by the $\eta-\eta^{\prime}$ pseudoscalar mixing angle $\phi$ in the flavor basis, phase space, and barrier penetration factors [15]. With $q$ being the two-body breakup momentum the amplitude ratio can be written as

$$
\frac{T_{J}^{\pi \eta^{\prime}}\left(m_{X}\right)}{T_{J}^{\pi \eta}\left(m_{X}\right)}=\tan \phi\left[\frac{q^{\pi \eta^{\prime}}\left(m_{X}\right)}{q^{\pi \eta}\left(m_{X}\right)}\right]^{J+1 / 2}
$$

Here simplified barrier factors of the form $q^{J}$ were used.

The bottom left plot in Fig. 3 shows in red the $D_{+}$intensity of the $\pi^{-} \eta$ channel scaled by the phase space factor from Eq. (2.1) overlaid on top of the corresponding intensity in the $\pi^{-} \eta^{\prime}$ final 

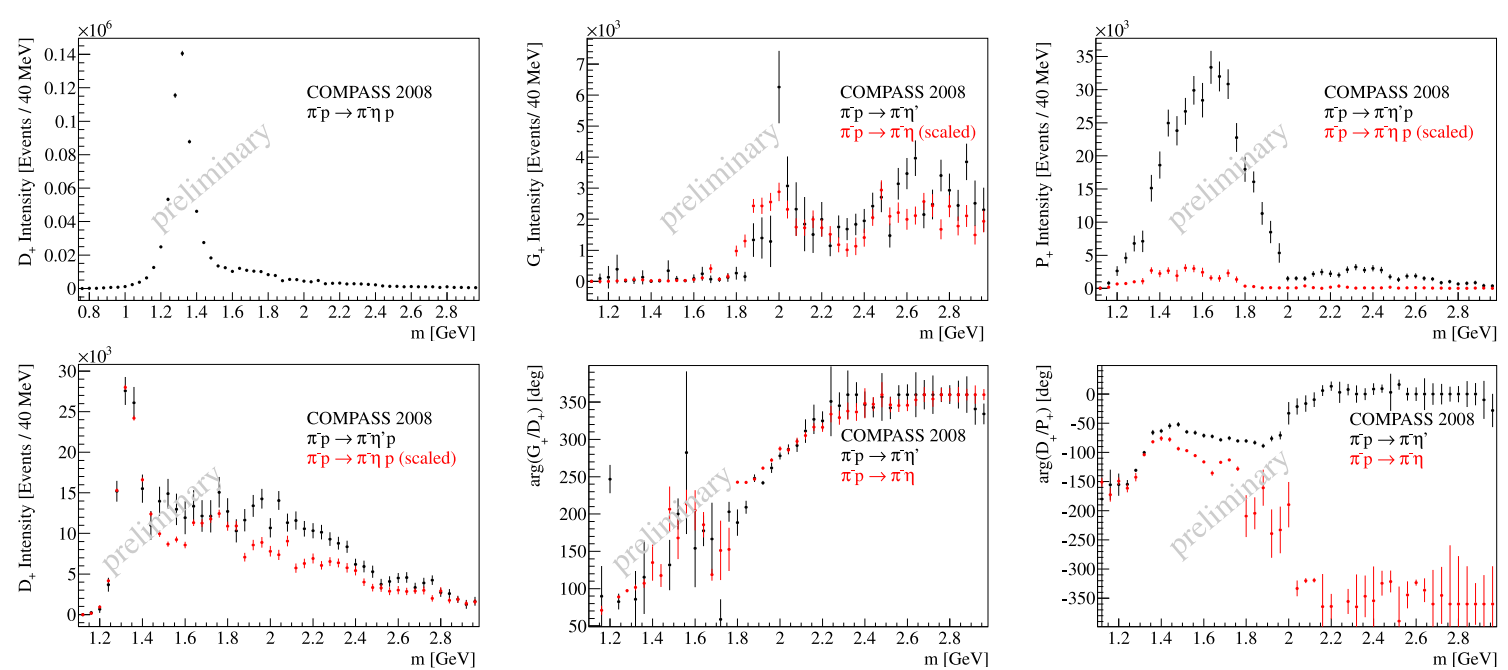

Figure 3: Left column: Intensity of the $2^{++} 1^{+}\left(D_{+}\right)$wave in the $\pi^{-} \eta$ (top) and $\pi^{-} \eta^{\prime}$ (bottom, black) channel, respectively. The red dots in the bottom plot show the $\pi^{-} \eta D_{+}$intensity scaled by phase space. Center and right column: Intensities and relative phases for $\pi^{-} \eta^{\prime}$ (black) and $\pi^{-} \eta$ scaled by phase space (red): $4^{++} 1^{+}\left(G_{+}\right)$intensity (top center), spin-exotic $1^{-+} 1^{+}\left(P_{+}\right)$intensity (top right), relative phases $G_{+}-D_{+}$(bottom center) and $D_{+}-P_{+}$(bottom right).

state. The phase space scaling leads to a remarkable similarity of the $\pi^{-} \eta$ and $\pi^{-} \eta^{\prime}$ intensities. This behavior is expected for light-quark resonances like the $a_{2}(1320)$, but it is rather astonishing that the scaling also holds in the high-mass region where non-resonant contributions are expected to dominate. As the middle row of Fig. 3 shows, the same behavior is observed for the $4^{++} 1^{+}$ $\left(G_{+}\right)$partial waves with the $a_{4}(2040)$. Also the relative phase between the $G_{+}$and the $D_{+}$waves is very similar in both channels which means that the physical composition of the two waves is almost identical.

The picture is much different for the spin-exotic $1^{-+} 1^{+}\left(P_{+}\right)$wave which is the dominant wave in the $\pi^{-} \eta^{\prime}$ channel with a prominent broad structure around $1.6 \mathrm{GeV} / c^{2}$ whereas it is strongly suppressed in the $\pi^{-} \eta$ channel (cf. Fig. 3 right). This observation is consistent with previous experiments and with the suspected non- $q \bar{q}$ character of this wave [16]. The $P_{+}$wave exhibits a slow phase motion with respect to the $D_{+}$wave in the $1.6 \mathrm{GeV} / c^{2}$ mass region which, however, evolves differently in the two final states. This is a hint that the resonant content is different. The resonance interpretation of the $P_{+}$wave requires a better understanding of the high-mass region of the $D_{+}$and $G_{+}$waves against which the phase motion of the $P_{+}$is measured. In this region nonresonant contributions from double-Regge processes are expected to play an important role which should be included in the fit model [17].

\section{$2.3 \pi^{-} \pi^{+} \pi^{-} \pi^{+} \pi^{-}$Final States from $\pi^{-}$Diffraction}

The diffractive reaction $\pi^{-} \mathrm{Pb} \rightarrow \pi^{-} \pi^{+} \pi^{-} \pi^{+} \pi^{-} \mathrm{Pb}$ is very interesting, because the $5 \pi$ final state gives access to the mass region around and above $2 \mathrm{GeV} / c^{2}$ which is also called the "lightmeson frontier". Only little is known about resonances in this region. There are many missing states as well as states that need confirmation.

A PWA of 200000 exclusive events was performed in the kinematic region $t^{\prime}<5 \cdot 10^{-3}(\mathrm{GeV} / c)^{2}$. Since a $\mathrm{Pb}$ target was used the recoil could not be detected. The partial-wave decomposition of 


\begin{tabular}{lll}
$\boldsymbol{\pi}^{+} \boldsymbol{\pi}^{-}$Isobars & $\boldsymbol{\pi}^{-} \boldsymbol{\pi}^{+} \boldsymbol{\pi}^{ \pm}$Isobars & $\boldsymbol{\pi}^{+} \boldsymbol{\pi}^{-} \boldsymbol{\pi}^{+} \boldsymbol{\pi}^{-}$Isobars \\
\hline$(\pi \boldsymbol{\pi})_{S \text {-wave }}, \rho(770)$ & $a_{1}(1260), a_{2}(1320)$ & $f_{2}(1270), f_{1}(1285), f_{0}(1370,1500), \rho^{\prime}(1450,1700)$
\end{tabular}

Table 1: Isobars used in the wave set of the $\pi^{-} \pi^{+} \pi^{-} \pi^{+} \pi^{-}$PWA [18].

diffractively produced $\pi^{-} \pi^{+} \pi^{-} \pi^{+} \pi^{-}$final states is challenging. Due to the lack of dominant structures in the $5 \pi$ invariant mass distribution and that of its subsystems, it is difficult to constraint the wave set which is potentially large, because of the many allowed isobars. In order to explore the model space more systematically an evolutionary algorithm was developed that uses a Bayesian goodnessof-fit criterion that takes into account the model complexity to iteratively find the best set of waves [18]. A pool of 284 waves was offered to the algorithm. The best model found consists of 31 partial wave plus an incoherent isotropic background wave. Table 1 lists the used isobars. An advantage of this automatized method is that it not only finds a wave set but also gives an estimate of the systematic uncertainty introduced by the particular choice of the wave set.

The mass dependence of a submatrix of the spin-density matrix consisting of 10 out of the 31 waves was parametrized using a simple model that consists of 6 resonances that were described by constant-width relativistic Breit-Wigners plus background terms. Although mixing and coupled-channel effects were neglected the model is able describe the data surprisingly well as is illustrated in Fig. 4. Table 2 summarizes the extracted resonance parameters. Being the first analysis of diffractively produced $\pi^{-} \pi^{+} \pi^{-} \pi^{+} \pi^{-}$, the results of this PWA should be interpreted with care. The analysis is still based on a number of assumptions. In particular

\begin{tabular}{|c|c|c|}
\hline Resonance & & $\begin{array}{r}\text { Fit result } \\
\mathrm{MeV} / c^{2}\end{array}$ \\
\hline \multirow[t]{2}{*}{$\pi(1300)$} & $M$ & 1400 (at limit) \\
\hline & $\Gamma$ & 500 (fixed) \\
\hline \multirow[t]{2}{*}{$\pi(1800)$} & $M$ & $1781=$ \\
\hline & $\Gamma$ & $168 \pm 9_{-15}^{+62}$ \\
\hline \multirow[t]{2}{*}{$a_{1}(1900)$} & $M$ & $1853 \pm 7_{-49}^{+36}$ \\
\hline & $\Gamma$ & $443 \pm 14_{-65}^{+98}$ \\
\hline \multirow[t]{2}{*}{$a_{1}(2200)$} & $M$ & $2202 \pm 8_{-11}^{+53}$ \\
\hline & $\Gamma$ & $402 \pm 17_{-51}^{+125}$ \\
\hline \multirow[t]{2}{*}{$\pi_{2}(1670)$} & $M$ & 1719.0 (fixed) \\
\hline & $\Gamma$ & 251.4 (fixed) \\
\hline \multirow[t]{2}{*}{$\pi_{2}(1880)$} & $M$ & $1854 \pm 6_{-9}^{+6}$ \\
\hline & $\Gamma$ & $259 \pm 13_{-31}^{+7}$ \\
\hline \multirow[t]{2}{*}{$\pi_{2}(2100)$} & $M$ & $2133 \pm 12_{-18}^{+43}$ \\
\hline & $\Gamma$ & $448 \pm 22_{-40}^{+80}$ \\
\hline
\end{tabular}

Table 2: Summary of extracted $5 \pi$ resonance parameters [18]. The first uncertainty is statistical, the second represents the systematic error including uncertainties from the choice of the final wave set.

the lack of knowledge on the properties of the four-pion isobars is difficult to quantify.

\section{Search for Scalar Glueball Candidates in Central Production}

Pomeron-Pomeron fusion processes are believed to provide a glue-rich environment which should lead to an enhanced cross section for the production of glueballs. Although glueball candidates are discussed in the literature, their existence could not yet be confirmed experimentally. Continuing the efforts of CERN OMEGA spectrometer in the late 1990s [19] COMPASS studies the central-production reaction $p p \rightarrow p_{\text {fast }} \pi^{+} \pi^{-} p_{\text {slow }}$ using a $190 \mathrm{GeV} / c$ positive secondary hadron beam which consists of $75 \% p, 24 \% \pi^{+}$, and $1 \% K^{+}$at the $\ell \mathrm{H}_{2}$ target [21].

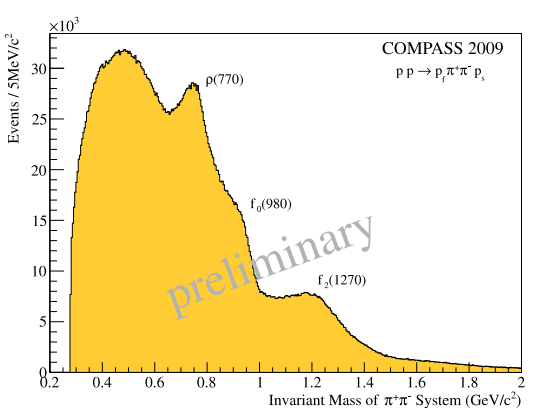

Figure 5: $\pi^{+} \pi^{-}$invariant mass distribution. 


$$
\begin{aligned}
& 0^{-+} \pi^{-} f_{0}(1500) S \\
& 0^{-+} \rho a_{1}(1260) S \\
& 1^{++} \pi^{-} f_{0}(1370) P \\
& 1^{++} \pi^{-} f_{1}(1285) P \\
& 1^{++} \rho \pi(1300) S \\
& 1^{++}(\pi \pi)_{S} a_{1} D \\
& 2^{-+} \pi^{-} f_{2}(1270) S \\
& 2^{-+} \rho a_{1}(1260) S \\
& 2^{-+} \rho a_{2}(1320) S \\
& 2^{-+} \rho a_{1}(1260) D
\end{aligned}
$$

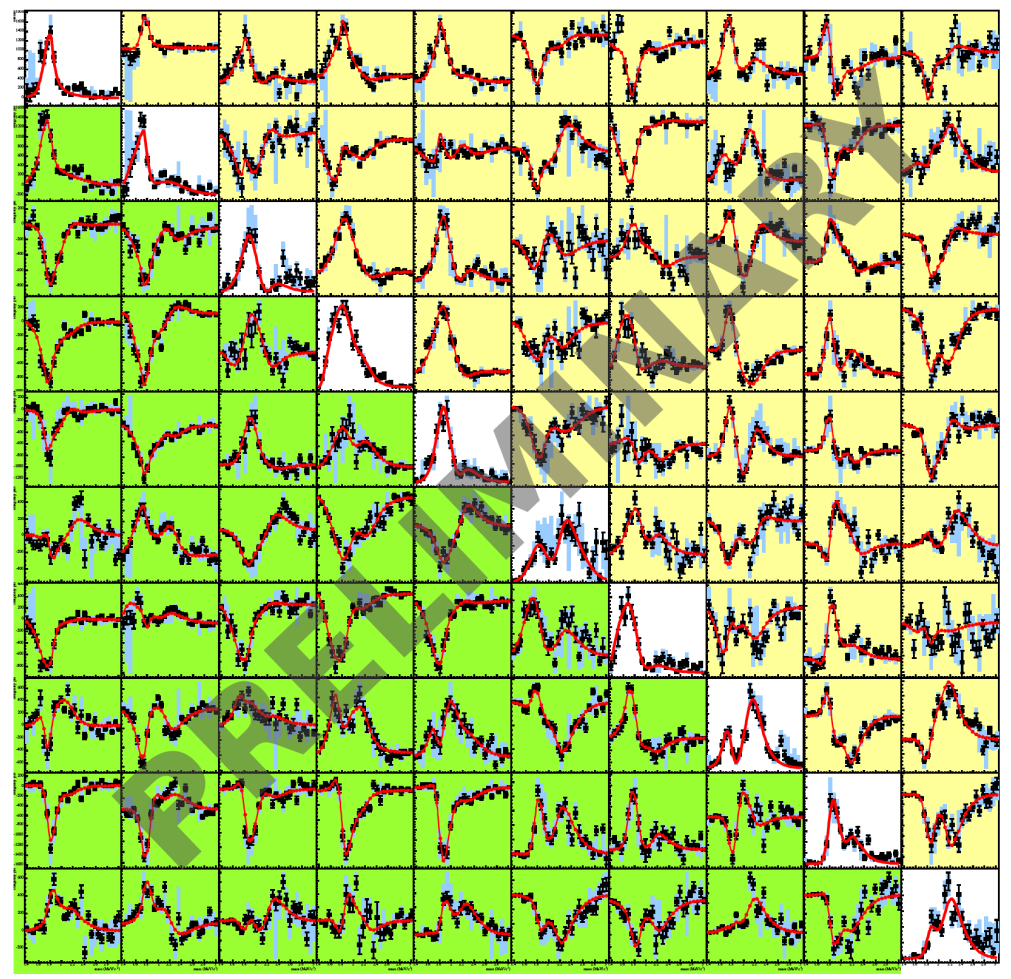

Figure 4: Fit of the $m_{5 \pi}$ dependence of a spin-density submatrix of 10 waves [18]. The diagonal elements show the wave intensities, the off-diagonal ones the interference terms (top right triangle: real, bottom left triangle: imaginary part). The light blue error bars represent the uncertainty from the choice of the wave set.

Centrally produced $\pi^{+} \pi^{-}$are separated from beam-

diffraction events by cutting on the invariant mass $m\left(p_{\text {fast }} \pi^{ \pm}\right)>1.5 \mathrm{GeV} / c^{2}$. Elastic scattering at the target vertex is ensured by the RPD trigger. This selects $\pi^{+} \pi^{-}$pairs within $\left|x_{F}\right| \leq 0.25$. At COMPASS energies in addition to Pomeron-Pomeron fusion also Reggeon-Pomeron and ReggeonReggeon processes are expected to contribute. This can be seen in the $\pi^{+} \pi^{-}$invariant mass spectrum shown in Fig. 5, where in addition to structures from $f_{0}(980)$ and $f_{2}(1270)$ a clear $\rho(770)$ peak is visible.

The employed PWA method is similar to the one used by WA102 [20]. The $\pi^{+} \pi^{-}$pair is assumed to be produced in the collision of two exchanged objects emitted by the scattered protons. The one emitted by the beam proton carries a squared four-momentum transfer $t_{1}$, the one from the target proton $t_{2}$, respectively. We make the strong assumption that exchange particle $t_{1}$ only transmits helicity $\lambda=0$ and that we can treat it like an external particle.

A complication arises from the fact that the two-pseudoscalar final state suffers from mathematical ambiguities, which means that for a given wave set different partial-wave decompositions result in exactly the same angular distribution. The PWA was performed using a wave set consisting of $S, P$, and $D$ waves which leads to eight ambiguous solutions. Additional constraints are needed to select the physical solution. For six solutions, most of the intensity accumulates in a single wave which is clearly unphysical, since at least one resonance is known to be present in $S$, $P$ and $D$ waves (cf. Fig. 5). The choice among the two remaining solutions is not evident, the intensities and phases are very similar. The remaining solutions are compatible with the physical 
constraints. A clear $f_{2}(1270)$ peak can be seen in the $2^{++} 0^{-}\left(D_{0}^{-}\right)$intensity. It is accompanied by a phase motion with respect to the $0^{++} 0^{-}\left(S_{0}^{-}\right)$wave. The $P$ waves show $\rho(770)$ peaks. The most interesting wave is, of course, the $S_{0}^{-}$. Its interpretation is, however, challenging and work in progress.

\section{Summary}

COMPASS is a unique apparatus to study light-quark hadron spectroscopy in diffractive and central production reactions. Large data sets were collected for various final states with charged as well as neutral particles using different target materials. The main focus of the first analyses lies on the search for spin-exotic mesons. $J^{P C}=1^{-+}$signals are observed in diffractively produced $3 \pi$ and $\pi \eta^{(\prime)}$ final states. However, their interpretation in terms of resonances is complicated by strong non-resonant contributions that need to be taken into account in the analysis. In the future, the search for spin-exotic signals will be extended to channels like $\pi^{-} \omega \pi^{0}$ and $\pi^{-}(K \bar{K})^{ \pm} \pi^{\mp}$. Glueball candidates are studied in centrally produced $\pi^{+} \pi^{-}$pairs. First PWA show promising results and this analysis is beeing extended to $K^{+} K^{-}, \pi^{0} \pi^{0}$, and $K_{S}^{0} \bar{K}_{S}^{0}$ final states.

\section{References}

[1] P. Abbon et al., Nucl. Instrum. Methods A577 (2007) 455; M. Alekseev et al., "The COMPASS 2008 Spectrometer" to be submitted to Nucl. Instrum. Methods A (2013).

[2] C. Meyer et al., Phys. Rev. C82 (2010) 025208; E. Klempt et al., Phys. Rept. 454 (2007) 1.

[3] A. Alekseev et al., Phys. Rev. Lett. 104 (2010) 241803.

[4] G. S. Adams et al., Phys. Rev. Lett. 81 (1998) 5760; Y. Khokhlov, Nucl. Phys. A663 (2000) 596; S. U. Chung et al., Phys. Rev. D65 (2002) 072001.

[5] J. D. Hansen et al., Nucl. Phys. B81 (1974) 403.

[6] S. U. Chung and T. L. Trueman, Phys. Rev. D11 (1975) 633.

[7] F. Haas, Proceedings of the XIV International Conference on Hadron Spectroscopy (hadron2011), Munich (2011), eConf C110613 (2011) [arXiv:1109.1789].

[8] F. von Hippel and C. Quigg, Phys. Rev. D5 (1972) 624.

[9] D.V. Amelin et al., Phys. Lett. B356 (1995) 595.

[10] G. Beladidze et al., Phys. Lett. B313, (1993) 276.

[11] A. Abele et al., Phys. Lett. B423, (1998) 175; D. Thompson et al., Phys. Rev. Lett. 79, (1997) 1630.

[12] E. I. Ivanov et al., Phys. Rev. Lett. 86, (2001) 3977.

[13] A. Szczepaniak, M. Swat, A. Dzierba, and S. Teige, Phys. Rev. Lett. 91, (2003) 092002.

[14] T. Schlüter, Proceedings of the Sixth International Conference on Quarks and Nuclear Physics (QNP2012), Paris (2012), PoS(QNP2012)074 [arXiv:1207.1076].

[15] A. Bramon, R. Escribano, and M. Scadron, Eur. Phys. J. C7 (1999) 271; T. Feldmann, P. Kroll, and B. Stech, Phys. Rev. D58 (1998) 114006; S. Okubo and K. Jagannathan, Phys. Rev. D15 (1977) 177.

[16] F. Close and H. Lipkin, Phys. Lett. B196 (1987) 245.

[17] V. Mathieu, these proceedings.

[18] S. Neubert, "First Amplitude Analysis of Resonant Structures in the 5-Pion Continuum at COMPASS”, PhD Thesis, TU München, 2012.

[19] A. Kirk, “The CERN OMEGA Spectrometer: 25 Year of Physics”, CERN 97-2 (1997) 31.

[20] D. Barberis et al., Phys. Lett. B453 (1999) 316.

[21] A. Austregesilo and T. Schlüter, Proceedings of the Sixth International Conference on Quarks and Nuclear Physics (QNP2012), Paris (2012), PoS(QNP2012)098 [arXiv:1207.0949]. 